\title{
Faith's Country Primitives: An Excel Project In Flexible Budgeting And Standard Cost Analysis
}

Katherine J. Silvester, Siena College, USA

\begin{abstract}
Cost accounting textbooks typically contain extensive technical coverage of standard costing and variance analysis in a manufacturing context. Covering the material adequately usually requires multiple class lectures, due to the complexity of the material. The calculations involved are detailed, multi-step, and can be numerically intensive. In order to make the material accessible to the learner, most textbook problems focus on the individual steps inherent in the budgeting, calculation, analysis, and interpretation process of standard costing and variance analysis. This simplification makes it feasible to deliver the material efficiently in the classroom. However, this pedagogical efficiency occurs at a cost, in that students may fail to understand the overall process and how the individual steps are interrelated.
\end{abstract}

This case has been used over the course of 10 semesters with over 500 students in a junior level Cost Accounting class for accounting majors. It has proven to be an effective tool for both reinforcing the variance analysis content that is delivered in the classroom, as well as for giving students an opportunity to practice and extend their Excel skills.

Keywords: Standard Costing; Excel; Variance Analysis

\section{INTRODUCTION}

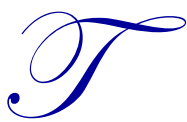

his paper presents an integrated Excel-based case that can be used in conjunction with a standard costing teaching module. The Excel-based case contains this Introduction and 4 case sections. The first case section contains the Student Case Description and Case Attachments. The second case section contains the Student Project Test Instructions and Data. The third section contains a printed copy of the Excel Spreadsheet Solutions to the Original Case and to the Project Test. The fourth and final case section contains the Teaching Notes and Suggestions for the Instructor. As noted later in the paper, an electronic version of the Excel spreadsheet, formulas, and solutions are also available to faculty upon request.

\section{SECTION 1: STUDENT CASE DESCRIPTION AND CASE ATTACHMENTS}

A. Project Overview. Faith's Country Primitives Company produces and sells fabric country primitive toys for use in home decor. Each toy uses cotton for the toy's clothing, fiber for the toy's outer shell, and stuffing for the toy's interior. You are the Controller for Faith's. As sales have grown, you have also become increasingly busy. To save yourself some time, you have decided to automate some of the reports that you have been producing manually every month. As a first step, you have decided to create an Excel spreadsheet program that your clerk can use to produce monthly variance reports for management.

Every month, your clerk will input new figures into your spreadsheet. However, your clerk isn't very knowledgeable about accounting, so he will need to depend on your Excel program to be accurate, complete, and understandable. Your clerk only knows how to input data, save files, and print in Excel. 
Your project is to create and produce three interconnected Standard Costing and Variance Analysis Reports in Excel. You must e-mail your Excel file to your professor by the Due Date. To demonstrate that your project works, you will also be required to test your project (Excel file) in a separately scheduled Project Test Session.

B. Creating the Reports. Creating your report will require you to create at least three worksheets in your Excel Workbook:

- Worksheet 1: Data Input Worksheet,

- Worksheet 2: A Flexible Budget Variance Report, and

- Worksheet 3: A Price and Efficiency Variance Report.

You will probably find it helpful to create additional Intermediary Worksheets to organize your calculations. However, these Intermediary Worksheets are not required.

Worksheet 1: The Report Name of Worksheet 1 is "Data Input Worksheet." Worksheet 1 should be a duplicate of the sample included as Attachment 1. Worksheet 1 should be formatted and highlighted as similarly as possible to the provided sample. Worksheet 1 serves as an input sheet for the rest of your Excel model. Every highlighted value is subject to change during the Project Test Session of your model. Data cannot be input into the other worksheets during the Project Test Session.

Your first worksheet must be used for data input only. Your second and third worksheets are reports for management. These latter reports must use the input data from the first worksheet, plus your Excel formulas, to generate a Flexible Budget Variance Analysis Report (Worksheet 2) and a Price and Efficiency Variance Report (Worksheet 3).

Therefore, your Workbook must be structured so that it will: (1) allow for any of the highlighted information on Worksheet 1 to be changed and (2) automatically adjust for these changes and correctly generate revised numbers, variance indicators, dates, etc. on the other two worksheets.

Rounding: Round all numbers on Worksheet 1 to the 3rd decimal place.

Worksheet 2: The Report Name of Worksheet 2 is "Flexible Budget Variance Report." Be sure to include a separate line item for each of the types of Direct Material for Faith's (Fiber, Stuffing, Cotton). See Attachment 2 for an outline of the format for this worksheet. ${ }^{1}$

Rounding: Round the "Standards" Column on Worksheet 2 to the $3^{\text {rd }}$ decimal place. Round the other columns on Worksheet 2 to the nearest whole dollar.

Worksheet 3: The Report Name of Worksheet 3 is "Price and Efficiency Variance Report." You must complete a table that organizes and reports the Price Variance, Efficiency Variance, and Flexible Budget Variances for each of the 6 different cost items (fiber, stuffing, cotton, labor, variable overhead, and fixed overhead). See Attachment 3 for an outline of the format for this worksheet.

Rounding: Round the variances on Worksheet 3 to the $3^{\text {rd }}$ decimal place.

Optional Additional Intermediary Worksheets. Do not show any additional or intermediary calculations or formulas on Worksheets 1, 2, or 3 of your Report, other than what is required. Management is already knowledgeable about how variances are calculated, and Management does not want to see a cluttered report.

\footnotetext{
${ }^{1}$ Horngren, Datar, and Rajan (2015) provide a thorough technical presentation of the material in Chapters 7 and 8 . An example of the basic format for a Flexible Budget Variance report is found on page 253.
} 
You may create additional optional worksheets to organize and calculate your variances or to organize intermediary figures. You may leave the optional worksheets in your workbook. These additional worksheets will not be graded; they will be for your use only.

Variance Absolute Values and Indicators. All variances must be reported as Absolute Values. You must also use the appropriate macros and/or "Nested If" commands to automatically indicate whether the variances are Favorable [F], Unfavorable [U], or neither (-), whenever variances are reported. Do not use colors to represent the Favorable or Unfavorable variances.

Attachment 1 Original Input Data

\section{Faith's Country Primitives Company Data Input Worksheet \\ January 2017}

Faith's Master Budget for Calendar Year

Selling Price

Expected Monthly Sales

Direct Materials

Standard Price of Cotton per yard

Standard Quantity of Cotton per toy

Standard Price of Fiber per yard

Standard Quantity of Fiber per toy

Standard Price of Stuffing per ounce

Standard Quantity of Stuffing per toy

Direct Labor

Standard Price of Labor

Stand Quantity of Labor per toy

Variable Manufacturing Overhead (in machine hours)

Standard Price

Standard Quantity

Fixed Overhead Per Month

Faith's Actual Results for

January 2017

Units Produced and Sold

Revenue

Direct Labor

Direct Materials - Cotton Purchased and Used

Direct Materials - Fiber Purchased and Used

Direct Materials - Stuffing Purchased and Used

Variable Overhead

Fixed Overhead
2017

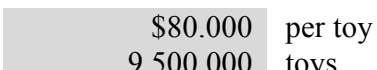

$9,500.000$ toys

$\$ 5.000$

1.850 yds.

$\$ 7.300$

2.250 yds

$\$ 0.220$

9.870 oz.

$\$ 15.250$ per hour

0.100 hours

$\$ 45.000$ per machine hour

0.300 machine hour(s) per toy

$\$ 120,000.000$ per month
$\$ 903.093 .750$

$\$ 17,230.000$

$\$ 101,574.000$

$\$ 192,235.810$

$\$ 20,964.440$

$21,500.000$ yds.

$119,094.000$ oz.

4,067.000 mach. hrs.
$\$ 149,600.000$ $\$ 124,500.000$ 


\begin{tabular}{|c|c|c|c|c|c|c|}
\hline & \multicolumn{4}{|c|}{$\begin{array}{c}\text { Faith's Country Primitives } \\
\text { Flexible Budget Variance Report } \\
\text { Month Year }\end{array}$} & \multirow[b]{2}{*}{ Static Budget } & \multirow[b]{2}{*}{ Standards } \\
\hline & $\begin{array}{l}\text { Actual } \\
\text { Results } \\
\end{array}$ & $\begin{array}{c}\text { Flexible Budget } \\
\text { Variances }\end{array}$ & $\begin{array}{c}\text { Flexible } \\
\text { Budget }\end{array}$ & $\begin{array}{c}\text { Sales Volume } \\
\text { Variances }\end{array}$ & & \\
\hline \multicolumn{7}{|l|}{ Units Sold } \\
\hline \multicolumn{7}{|l|}{ Revenue } \\
\hline \multicolumn{7}{|l|}{ Variable Costs } \\
\hline \multicolumn{7}{|l|}{ Direct Materials-Cotton } \\
\hline \multicolumn{7}{|l|}{ Direct Materials-Fiber } \\
\hline \multicolumn{7}{|l|}{ Direct Materials-Stuffing } \\
\hline \multicolumn{7}{|l|}{ Direct Mfg. Labor } \\
\hline \multicolumn{7}{|l|}{ Variable Mfg. Overhead } \\
\hline \multicolumn{7}{|l|}{ Total Variable Costs } \\
\hline \multicolumn{7}{|l|}{ Contribution Margin } \\
\hline \multicolumn{7}{|l|}{ Fixed Manufacturing Costs } \\
\hline Operating Income & & & & & & \\
\hline
\end{tabular}

\begin{tabular}{|l|c|c|c|}
\hline \multicolumn{3}{|c|}{$\begin{array}{c}\text { Faith's Country Primitives } \\
\text { Price and Efficiency Variance Report } \\
\text { Month } 3\end{array}$} \\
\hline & Price or Spending Variance & Efficiency Variance & Flexible Budget Variance \\
\hline Direct Material-Cotton & & & \\
Direct Material-Fiber & & & \\
Direct Material-Stuffing & & & \\
Direct Labor & & & \\
Variable Overhead & & & \\
Fixed Overhead & & & \\
\hline
\end{tabular}

\section{SECTION 2: STUDENT PROJECT TEST INSTRUCTIONS AND DATA}

There will be a formal Student Project Test Session scheduled in a Computer Lab. During the Project Test Session, the following will be expected.

- You must load the same Excel file that was submitted to your professor.

- You must print each of the Original Worksheets 1,2, and 3 on separate pages.

- You will be given revised test input data and dates for the highlighted information on Worksheet 1. (Note that the changed information is "boxed.")

- You must change the highlighted information on Worksheet 1, as indicated. Worksheets 2 and 3 must then automatically and correctly generate revised numbers and dates.

- You will print each of the Revised Worksheets 1, 2, and 3 on separate pages. 
Remember that you cannot input any numbers directly into Worksheets 2 and 3 during this Test Session. Therefore, your Original Project must include formulas that will automatically use the revised Test data from Worksheet 1 to generate the correct figures and dates for Worksheets 2 and 3.

Attachment 4 Project Test Input Data

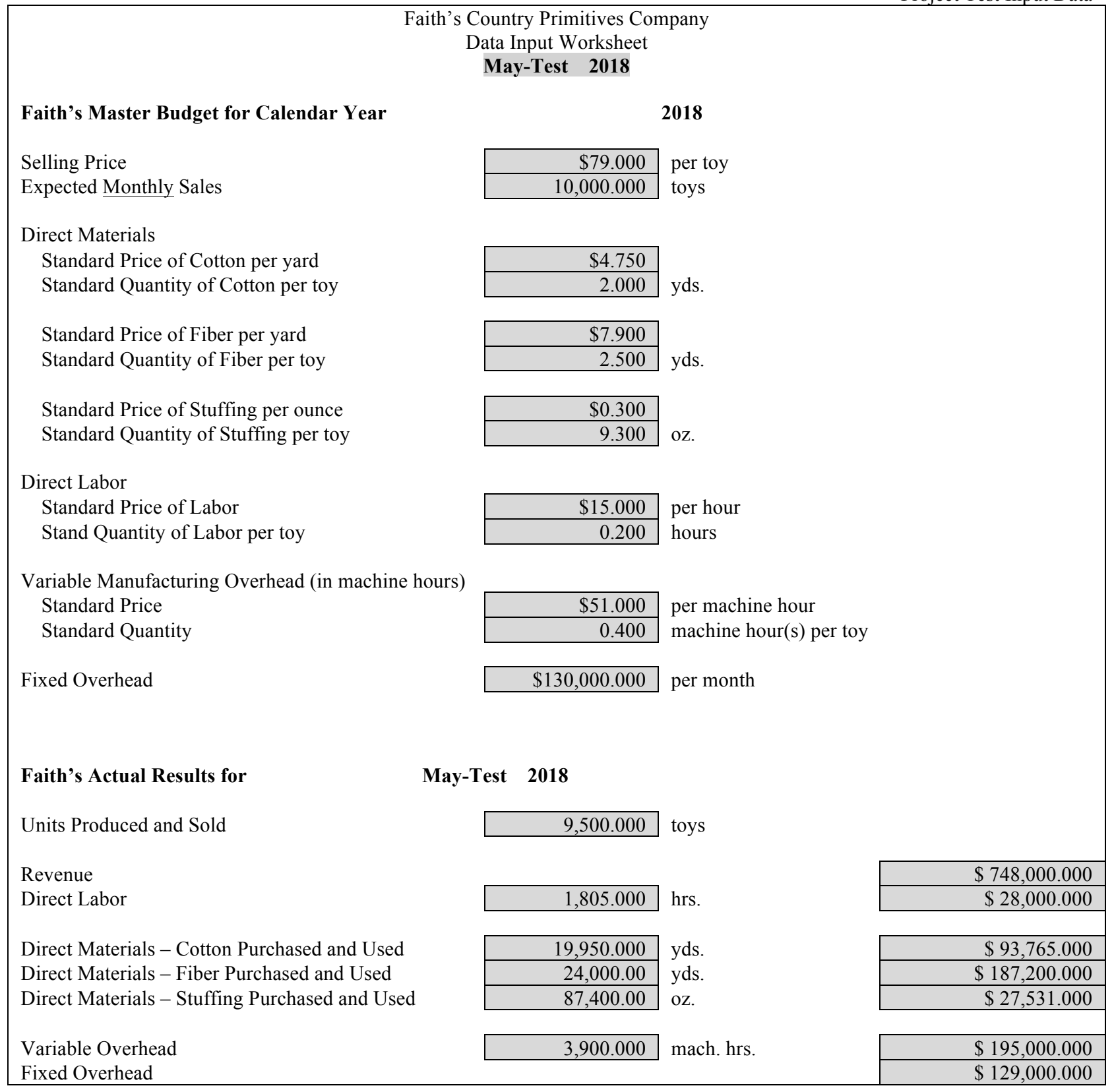

\section{SECTION 3: EXCEL SPREADSHEET SOLUTIONS TO THE ORIGINAL CASE AND TO THE PROJECT TEST}

The Excel-generated solutions to the Original Case are provided as Exhibits 1,2 and 3. Exhibit 3 contains an optional Intermediary Worksheet that students might use to organize their intermediary work product. 
Exhibits 4, 5, and 6 contain the solutions to the Project Test, as well as an optional Intermediary Worksheet.

All of the Excel solution files (with formulas) are available to other faculty by contacting the author by e-mail (ksilvester@siena.edu) and providing verification of college affiliation.

Exhibit 1

Solution - Original Project Data

\begin{tabular}{|c|c|c|c|c|c|c|c|c|c|c|c|c|c|}
\hline \multicolumn{14}{|c|}{$\begin{array}{c}\text { Faith's Country Primitives } \\
\text { Flexible Budget Variance Report } \\
\text { January } 2017 \\
\end{array}$} \\
\hline & \multirow{2}{*}{\multicolumn{2}{|c|}{$\begin{array}{l}\text { Actual } \\
\text { Results } \\
11,115 \\
\end{array}$}} & \multicolumn{3}{|c|}{$\begin{array}{c}\text { Flexible Budget } \\
\text { Variances }\end{array}$} & \multirow{2}{*}{\multicolumn{2}{|c|}{$\begin{array}{c}\begin{array}{c}\text { Flexible } \\
\text { Budget }\end{array} \\
11,115 \\
\end{array}$}} & \multicolumn{2}{|c|}{$\begin{array}{c}\text { Sales Volume } \\
\text { Variances }\end{array}$} & \multicolumn{2}{|c|}{ Static Budget } & \multicolumn{2}{|c|}{ Standards } \\
\hline Units Sold & & & & 0 & - & & & 1,615 & $\mathrm{~F}$ & & 9,500 & & 1 \\
\hline Revenue & $\$$ & 903,094 & $\$$ & 13,894 & $\mathrm{~F}$ & $\$$ & 889,200 & $\$ 129,200$ & $\mathrm{~F}$ & $\$$ & 760,000 & $\$$ & 80.00 \\
\hline Variable Costs & & & & & & & & & & & & & \\
\hline Direct Materials-Cotton & $\$$ & 101,574 & $\$$ & 1,240 & $\mathrm{~F}$ & $\$$ & 102,814 & $\$ 14,939$ & $\mathrm{U}$ & $\$$ & 87,875 & $\$$ & 9.250 \\
\hline Direct Materials-Fiber & $\$$ & 192,236 & $\$$ & 9,672 & $\mathrm{U}$ & $\$$ & 182,564 & $\$ 26,526$ & $\mathrm{U}$ & $\$$ & 156,038 & $\$$ & 16.425 \\
\hline Direct Materials-Stuffing & $\$$ & 20,964 & $\$$ & 3,171 & $\mathrm{~F}$ & $\$$ & 24,135 & $\$ 3,507$ & $\mathrm{U}$ & $\$$ & 20,628 & $\$$ & 2.171 \\
\hline Direct Mfg. Labor & $\$$ & 17,230 & $\$$ & 280 & $\mathrm{U}$ & $\$$ & 16,950 & 2,463 & $\mathrm{U}$ & $\$$ & 14,488 & $\$$ & 1.525 \\
\hline Variable Mfg. Overhead & $\$$ & 149,600 & $\$$ & 453 & $\mathrm{~F}$ & $\$$ & 150,053 & $\$ 21,803$ & $\mathrm{U}$ & $\$$ & 128,250 & $\$$ & 13.500 \\
\hline Total Variable Costs & $\$$ & 481,604 & $\$$ & 5,089 & $\mathrm{U}$ & $\$$ & 476,516 & $\$ 69,237$ & $\mathrm{U}$ & $\$$ & 407,278 & $\$$ & 42.871 \\
\hline Contribution Margin & $\$$ & 421,490 & $\$$ & 8,805 & $\mathrm{~F}$ & $\$$ & 412,684 & $\$ 59,963$ & $\mathrm{~F}$ & $\$$ & 352,722 & $\$$ & 37.129 \\
\hline Fixed Manufacturing Costs & $\$$ & 124,500 & $\underline{\$}$ & 4,500 & $\mathrm{U}$ & $\$$ & 120,000 & 0 & - & $\$$ & 120,000 & & \\
\hline Operating Income & $\$$ & 296,990 & $\$$ & 4,305 & $\mathrm{~F}$ & $\$$ & 292,684 & $\$ 59,963$ & $\mathrm{~F}$ & $\$$ & 232,722 & & \\
\hline
\end{tabular}

Exhibit 2

Solution - Original Project Data

\begin{tabular}{|c|c|c|c|c|c|c|c|c|c|}
\hline \multicolumn{10}{|c|}{$\begin{array}{c}\text { Faith's Country Primitives } \\
\text { Price and Efficiency Variance Report } \\
\text { January } 2017\end{array}$} \\
\hline & \multicolumn{3}{|c|}{ Price or Spending Variance } & \multicolumn{3}{|c|}{ Efficiency Variance } & \multicolumn{3}{|c|}{ Flexible Budget Variance } \\
\hline Direct Material-Cotton & $\$$ & $10,459.00$ & U & $\$$ & $11,698.750$ & $\mathrm{~F}$ & $\$$ & $1,239.750$ & $\mathrm{~F}$ \\
\hline Direct Material-Fiber & $\$$ & $35,285.810$ & $\mathrm{U}$ & $\$$ & $25,613.875$ & $\mathrm{~F}$ & $\$$ & $9,671.935$ & $\mathrm{U}$ \\
\hline Direct Material-Stuffing & $\$$ & $5,236.240$ & $\mathrm{~F}$ & $\$$ & $2,065.569$ & $\mathrm{U}$ & $\$$ & $3,170.671$ & $\mathrm{~F}$ \\
\hline Direct Labor & $\$$ & $1,915.950$ & $\mathrm{U}$ & $\$$ & $1,636.325$ & $\mathrm{~F}$ & $\$$ & 279.625 & $\mathrm{U}$ \\
\hline Variable Overhead & $\$$ & $33,415.00$ & $\mathrm{~F}$ & $\$$ & $32,962.500$ & U & $\$$ & 452.500 & $\mathrm{~F}$ \\
\hline Fixed Overhead & $\$$ & $4,500.00$ & $\mathrm{U}$ & $\$$ & - & - & $\$$ & $4,500.000$ & $\mathrm{U}$ \\
\hline
\end{tabular}




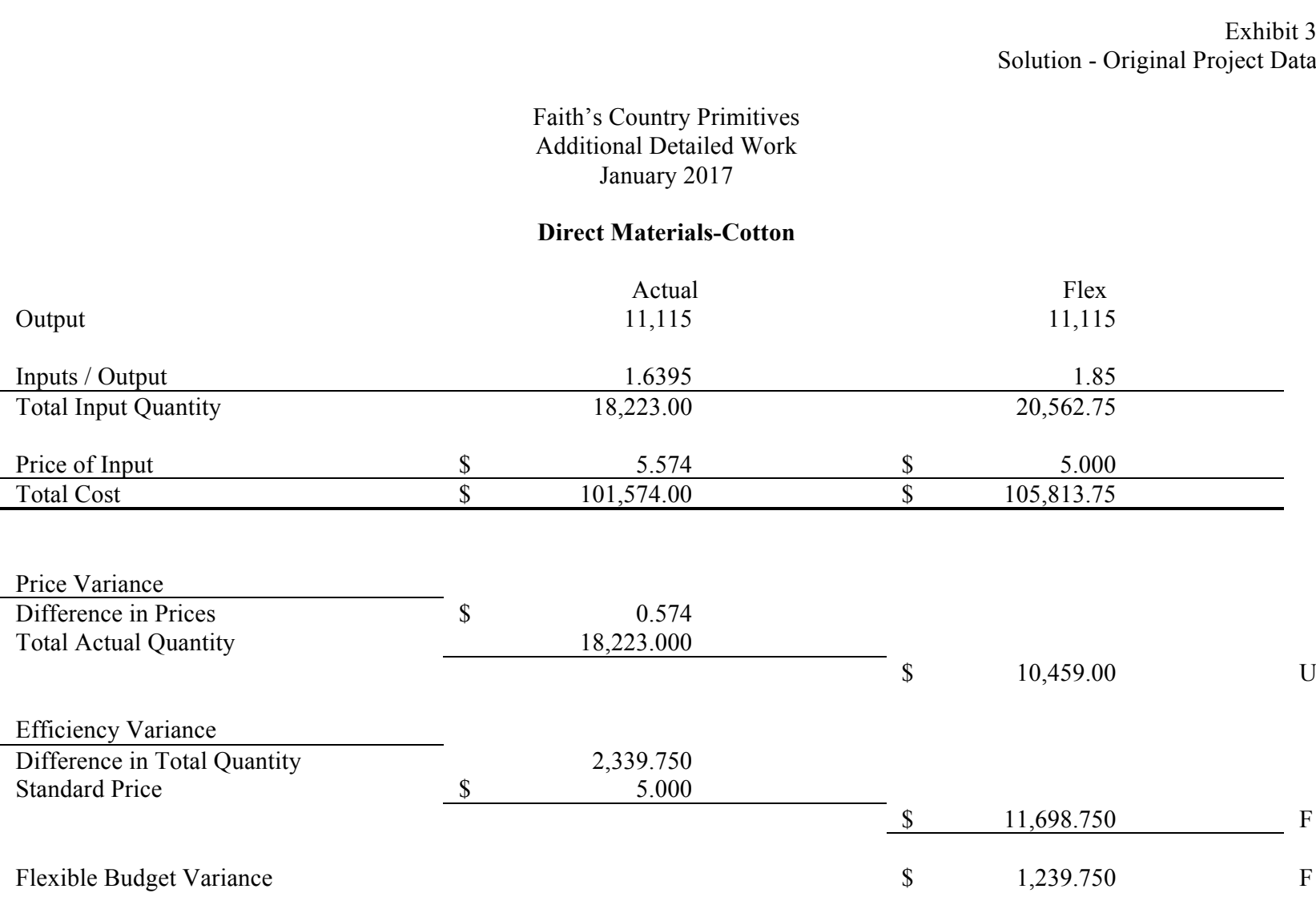

Direct Materials-Fiber

\begin{tabular}{|c|c|c|c|c|}
\hline Output & & $\begin{array}{c}\text { Actual } \\
11,115\end{array}$ & & $\begin{array}{c}\text { Flex } \\
11,115\end{array}$ \\
\hline Inputs / Output & & 1.9343 & & 2.25 \\
\hline Total Input Quantity & & $21,500.000$ & & $25,008.75$ \\
\hline Price of Input & $\$$ & 8.941 & $\$$ & 7.30 \\
\hline Total Cost & $\$$ & $192,235.81$ & $\$$ & $182,563.88$ \\
\hline
\end{tabular}

\begin{tabular}{l} 
Price Variance \\
\hline Difference in Prices \\
Total Actual Quantity \\
Efficiency Variance
\end{tabular}

$\$ \quad 1.641$
$21,500.000$

$\$ \quad 35,285.810$

Difference in Total Quantity

Standard Price

Flexible Budget Variance

\begin{tabular}{rr} 
& $3,508.750$ \\
$\$$ & 7.300 \\
\hline
\end{tabular}

$\$$

$\$$
$25,613.875$

$\mathrm{F}$

$9,671.935$

U 


\section{Direct Materials-Stuffing}

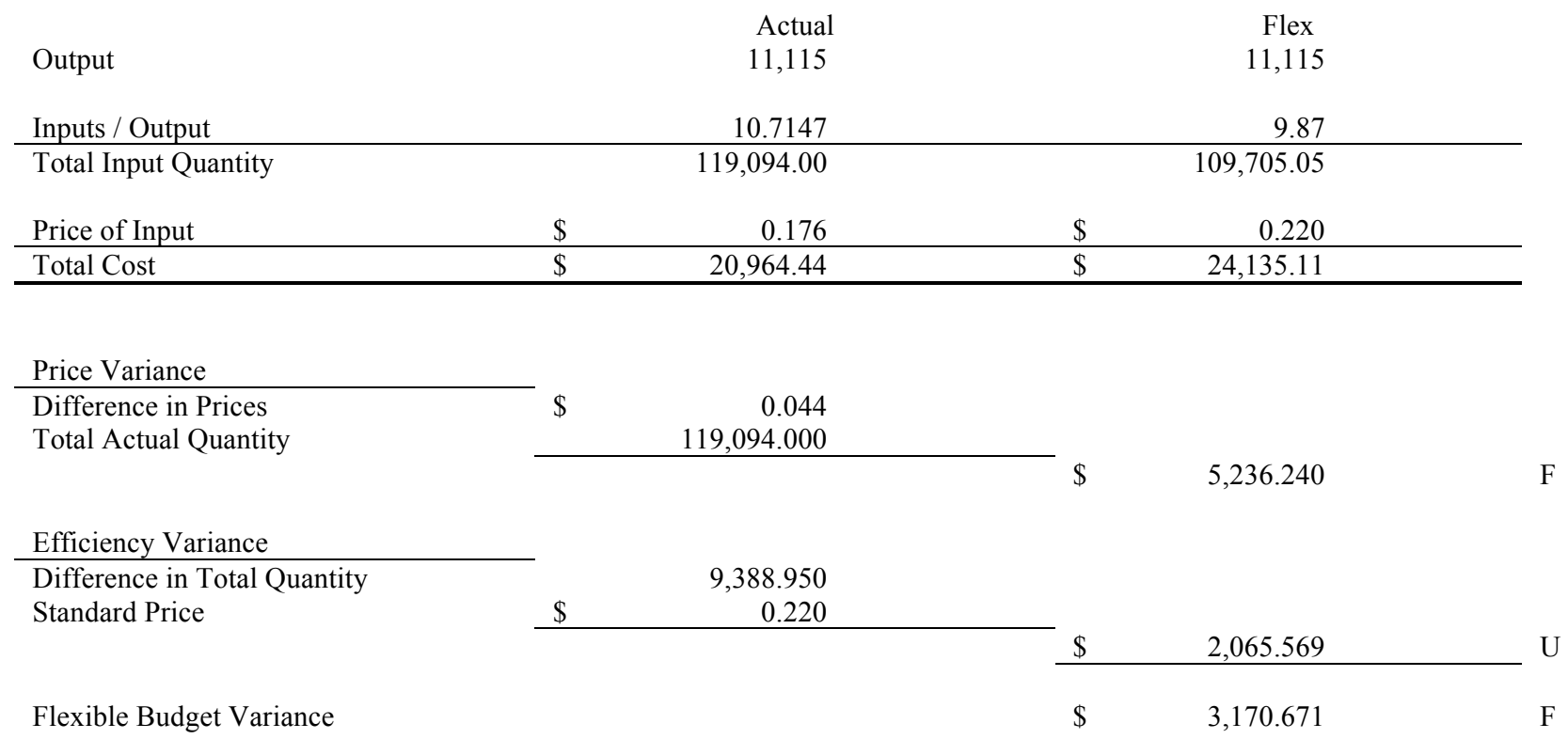

\section{Direct Labor}

\begin{tabular}{|c|c|c|c|}
\hline Output & & $\begin{array}{c}\text { Actual } \\
11,115\end{array}$ & $\begin{array}{c}\text { Flex } \\
11,115\end{array}$ \\
\hline Inputs / Output & & 0.0903 & 0.100 \\
\hline Total Input Quantity & & $1,004.20$ & $1,111.50$ \\
\hline Price of Input & $\$$ & 17.158 & 15.250 \\
\hline Total Cost & $\$$ & $17,230.00$ & $16,950.38$ \\
\hline
\end{tabular}

Price Variance

Difference in Prices

$\$ \quad 1.908$

Total Actual Quantity $1,004.200$

\$ $\quad 1,915.950$

$\mathrm{U}$

Efficiency Variance

Difference in Total Quantity

107.300

Standard Price

$\$$

15.250

Flexible Budget Variance

$\$$

$1,636.325$

F

279.625

$\mathrm{U}$ 


\section{Variable Overhead}

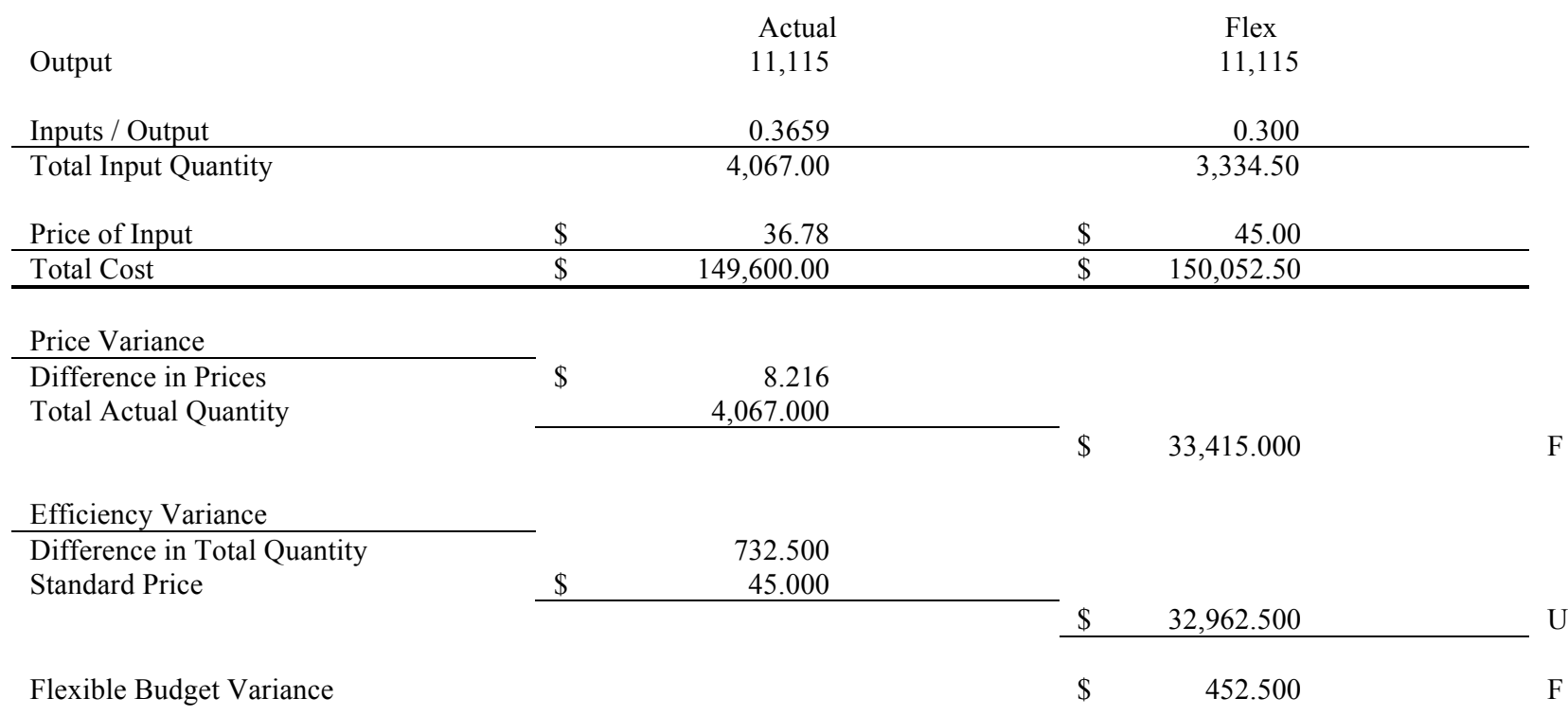

\section{Fixed Overhead}

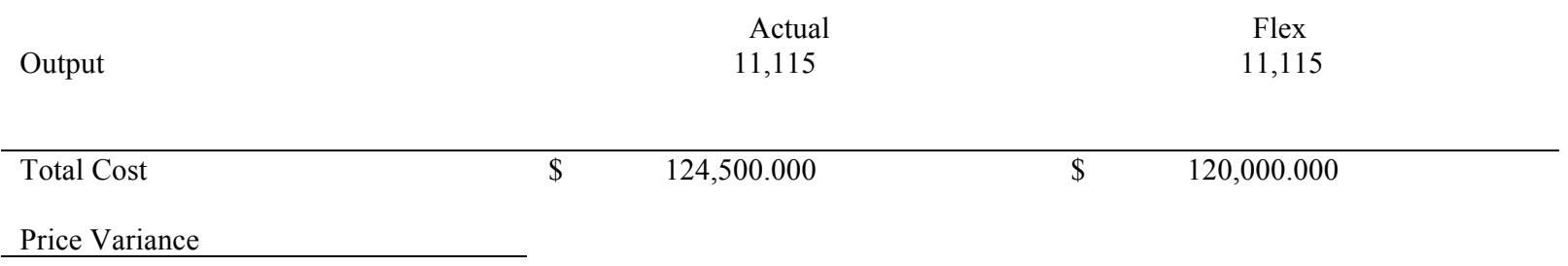

Efficiency Variance 
Exhibit 4

Solution - Project Test

\begin{tabular}{|c|c|c|c|c|c|c|c|c|c|c|c|c|c|}
\hline \multicolumn{14}{|c|}{$\begin{array}{c}\text { Faith's Country Primitives } \\
\text { Flexible Budget Variance Report } \\
\text { May-Test } 2018\end{array}$} \\
\hline & \multicolumn{2}{|r|}{$\begin{array}{l}\text { Actual } \\
\text { Results }\end{array}$} & \multicolumn{3}{|c|}{$\begin{array}{c}\text { Flexible Budget } \\
\text { Variances }\end{array}$} & \multicolumn{2}{|r|}{$\begin{array}{c}\text { Flexible } \\
\text { Budget }\end{array}$} & \multicolumn{2}{|c|}{$\begin{array}{l}\text { Sales Volume } \\
\text { Variances }\end{array}$} & \multicolumn{2}{|c|}{ Static Budget } & \multicolumn{2}{|c|}{ Standards } \\
\hline Units Sold & & 9,500 & & 0 & - & & 9,500 & 500 & $\mathrm{U}$ & & 10,000 & & 1 \\
\hline Revenue & $\$$ & 748,000 & $\$$ & 2,500 & $\mathrm{U}$ & $\$$ & 750,500 & $\$ 39,500$ & $\mathrm{U}$ & $\$$ & 790,000 & $\$$ & 79.000 \\
\hline \multicolumn{14}{|l|}{ Variable Costs } \\
\hline Direct Materials-Cotton & $\$$ & 93,765 & $\$$ & 3,515 & $\mathrm{U}$ & $\$$ & 90,250 & $\$ 4,750$ & $\mathrm{~F}$ & $\$$ & 95,000 & $\$$ & 9.500 \\
\hline Direct Materials-Fiber & $\$$ & 187,200 & $\$$ & 425 & $\mathrm{~F}$ & $\$$ & 187,625 & $\$ 9,875$ & $\mathrm{~F}$ & $\$$ & 197,500 & $\$$ & 19.750 \\
\hline Direct Materials-Stuffing & $\$$ & 27,531 & $\$$ & 1,026 & $\mathrm{U}$ & $\$$ & 26,505 & $\$ 1,395$ & $\mathrm{~F}$ & $\$$ & 27,900 & $\$$ & 2.790 \\
\hline Direct Mfg. Labor & $\$$ & 28,000 & $\$$ & 500 & $\mathrm{~F}$ & $\$$ & 28,500 & $\$ 1,500$ & $\mathrm{~F}$ & $\$$ & 30,000 & $\$$ & 3.000 \\
\hline Variable Mfg. Overhead & $\$$ & 195,000 & $\$$ & 1,200 & $\mathrm{U}$ & $\$$ & 193,800 & $\$ 10,200$ & $\mathrm{~F}$ & $\$$ & 204,000 & $\$$ & 20.400 \\
\hline Total Variable Costs & $\$$ & 531,496 & $\$$ & 4,816 & $\mathrm{U}$ & $\$$ & 526,680 & $\$ 27,720$ & $\mathrm{~F}$ & $\$$ & 554,400 & $\$$ & 55.440 \\
\hline Contribution Margin & $\$$ & 216,504 & $\$$ & 7,316 & $\mathrm{U}$ & $\$$ & 223,820 & $\$ 11,780$ & U & $\$$ & 235,600 & $\$$ & 23.560 \\
\hline Fixed Manufacturing Costs & $\$$ & 129,000 & $\$$ & 1,000 & $\mathrm{~F}$ & $\$$ & 130,000 & 0 & - & $\$$ & 130,000 & & \\
\hline Operating Income & $\$$ & 87,504 & $\$$ & 6,316 & $\mathrm{U}$ & $\$$ & 93,820 & $\$ 11,780$ & $\mathrm{U}$ & $\$$ & 105,600 & & \\
\hline
\end{tabular}

Exhibit 5 Solution - Project Test

\begin{tabular}{|c|c|c|c|c|c|c|c|c|c|}
\hline \multicolumn{10}{|c|}{$\begin{array}{c}\text { Faith's Country Primitives } \\
\text { Price and Efficiency Variance Report } \\
\text { May-Test } 2018\end{array}$} \\
\hline & \multicolumn{3}{|c|}{$\begin{array}{c}\text { Price or Spending } \\
\text { Variance }\end{array}$} & \multicolumn{3}{|c|}{ Efficiency Variance } & \multicolumn{3}{|c|}{ Flexible Budget Variance } \\
\hline Direct Material-Cotton & $\$$ & 997.500 & $\mathrm{~F}$ & $\$$ & $4,512.500$ & $\mathrm{U}$ & $\$$ & $3,515.000$ & $\mathrm{U}$ \\
\hline Direct Material-Fiber & $\$$ & $2,400.000$ & $\mathrm{~F}$ & $\$$ & $1,975.000$ & $\mathrm{U}$ & $\$$ & 425.000 & $\mathrm{~F}$ \\
\hline Direct Material-Stuffing & $\$$ & $1,311.000$ & $\mathrm{U}$ & $\$$ & 285.000 & $\mathrm{~F}$ & $\$$ & $1,026.000$ & $\mathrm{U}$ \\
\hline Direct Labor & $\$$ & 925.000 & $\mathrm{U}$ & $\$$ & $1,425.000$ & $\mathrm{~F}$ & $\$$ & 500.000 & $\mathrm{~F}$ \\
\hline Variable Overhead & $\$$ & $3,900.000$ & $\mathrm{~F}$ & $\$$ & $5,100.000$ & $\mathrm{U}$ & $\$$ & $1,200.00$ & $\mathrm{U}$ \\
\hline Fixed Overhead & $\$$ & $1,000.000$ & $\mathrm{~F}$ & $\$$ & - & - & $\$$ & $1,000.000$ & $\mathrm{~F}$ \\
\hline
\end{tabular}




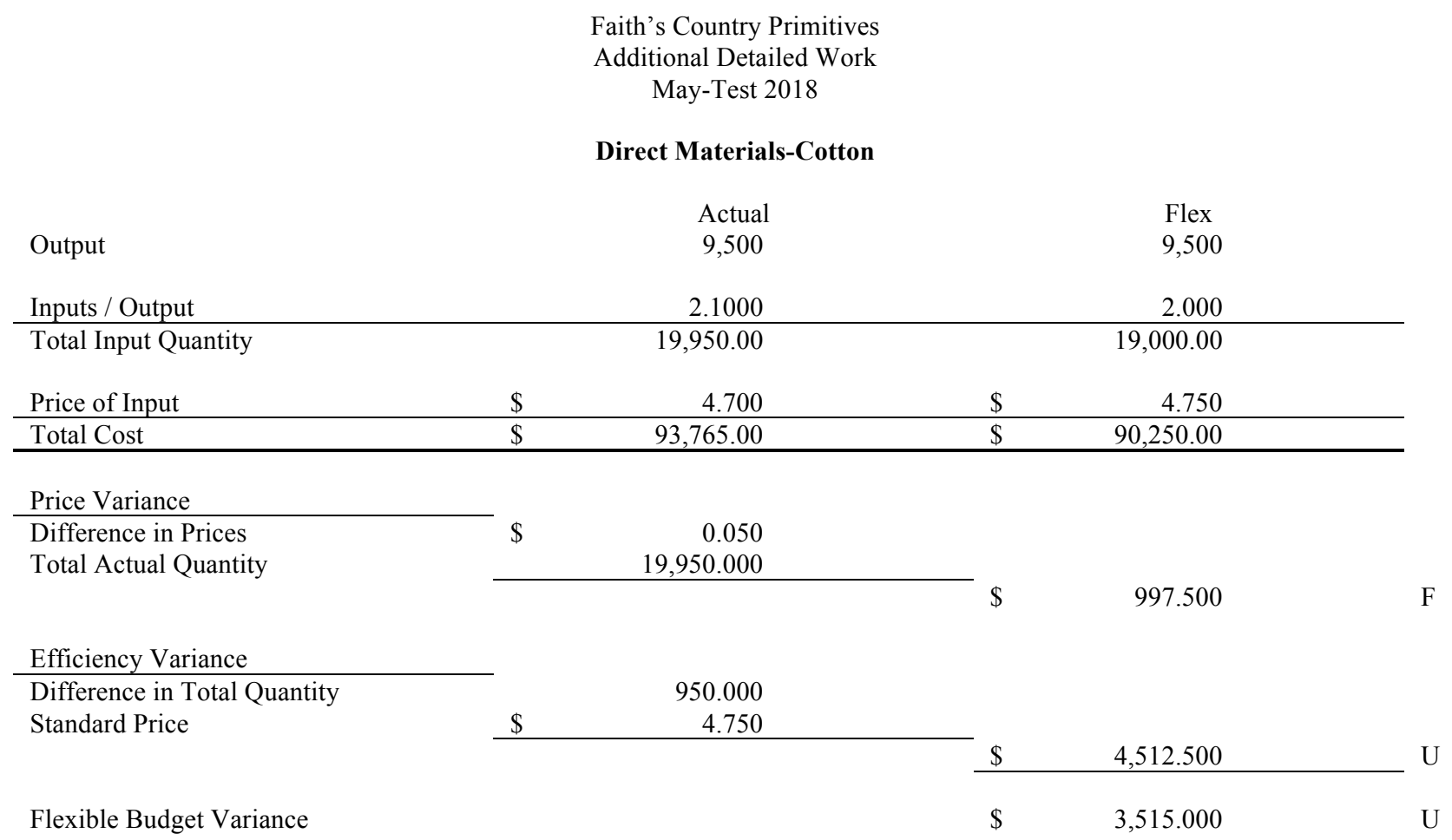

\section{Direct Materials-Fiber}

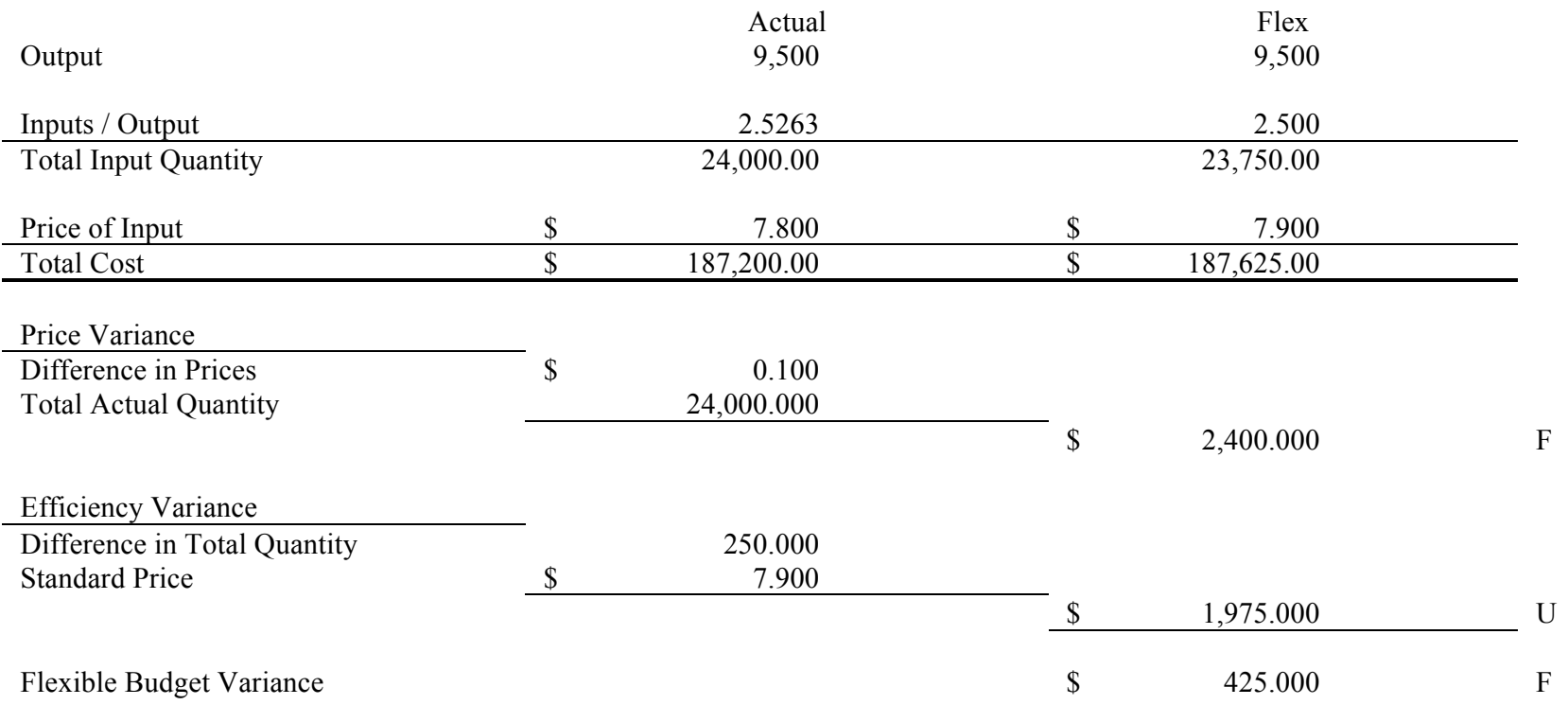


Direct Materials-Stuffing

\begin{tabular}{|c|c|c|c|c|}
\hline & & Actual & & Flex \\
\hline Inputs / Output & & 9.200 & & 9.300 \\
\hline Total Input Quantity & & $87,400.00$ & & $88,350.00$ \\
\hline Price of Input & $\$$ & 0.315 & $\$$ & 0.300 \\
\hline Total Cost & $\$$ & $27,531.00$ & $\$$ & $26,505.00$ \\
\hline \multicolumn{5}{|l|}{ Price Variance } \\
\hline \multirow{2}{*}{$\begin{array}{l}\text { Difference in Prices } \\
\text { Total Actual Quantity }\end{array}$} & $\$$ & $\begin{array}{r}0.015 \\
87400000\end{array}$ & \multirow[b]{2}{*}{$\$$} & \multirow[b]{2}{*}{$1,311.000$} \\
\hline & & & & \\
\hline \multicolumn{5}{|l|}{ Efficiency Variance } \\
\hline Difference in Total Quantity & & 950.000 & & \\
\hline \multirow[t]{2}{*}{ Standard Price } & $\$$ & 0.300 & & \\
\hline & & & $\$$ & 285.000 \\
\hline Flexible Budget Variance & & & $\$$ & $1,026.000$ \\
\hline
\end{tabular}

\section{Direct Labor}

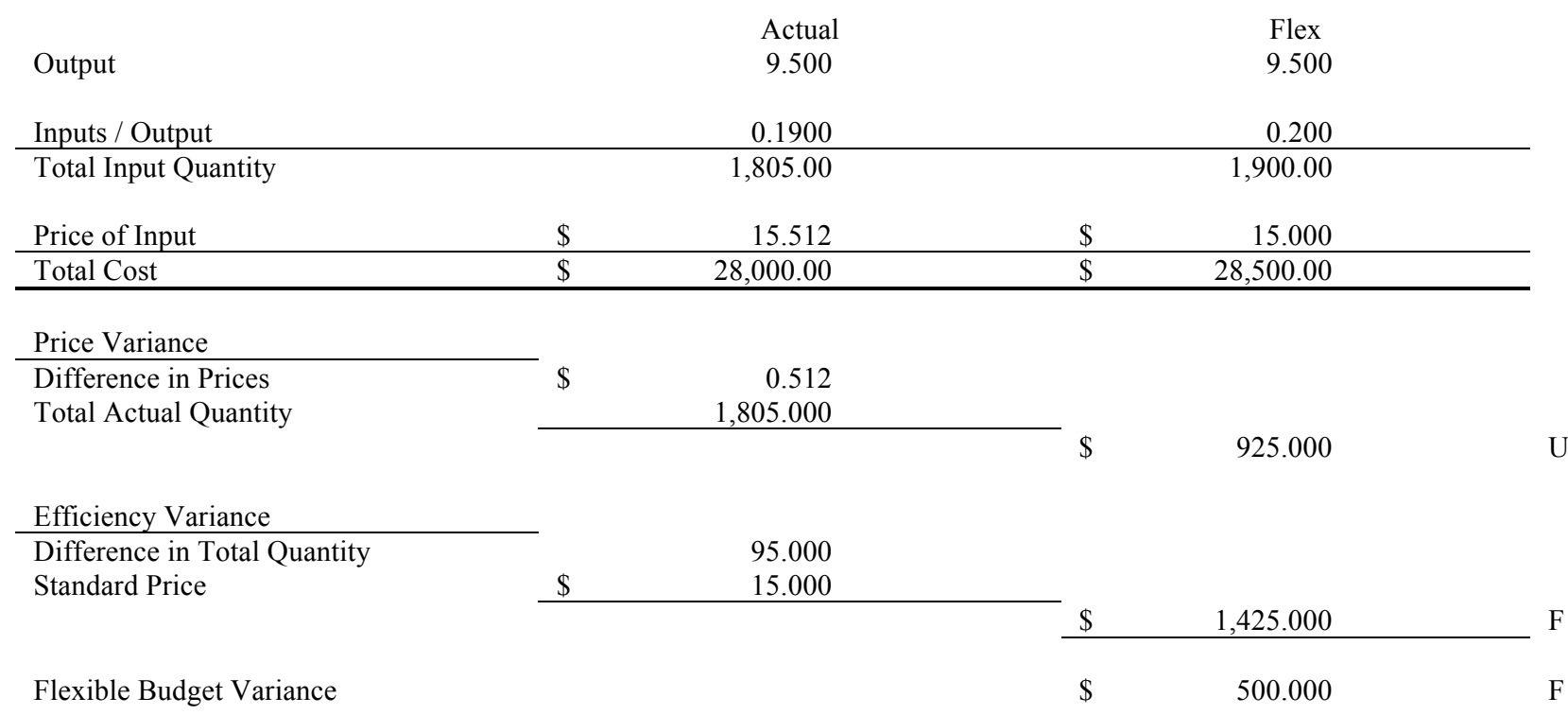




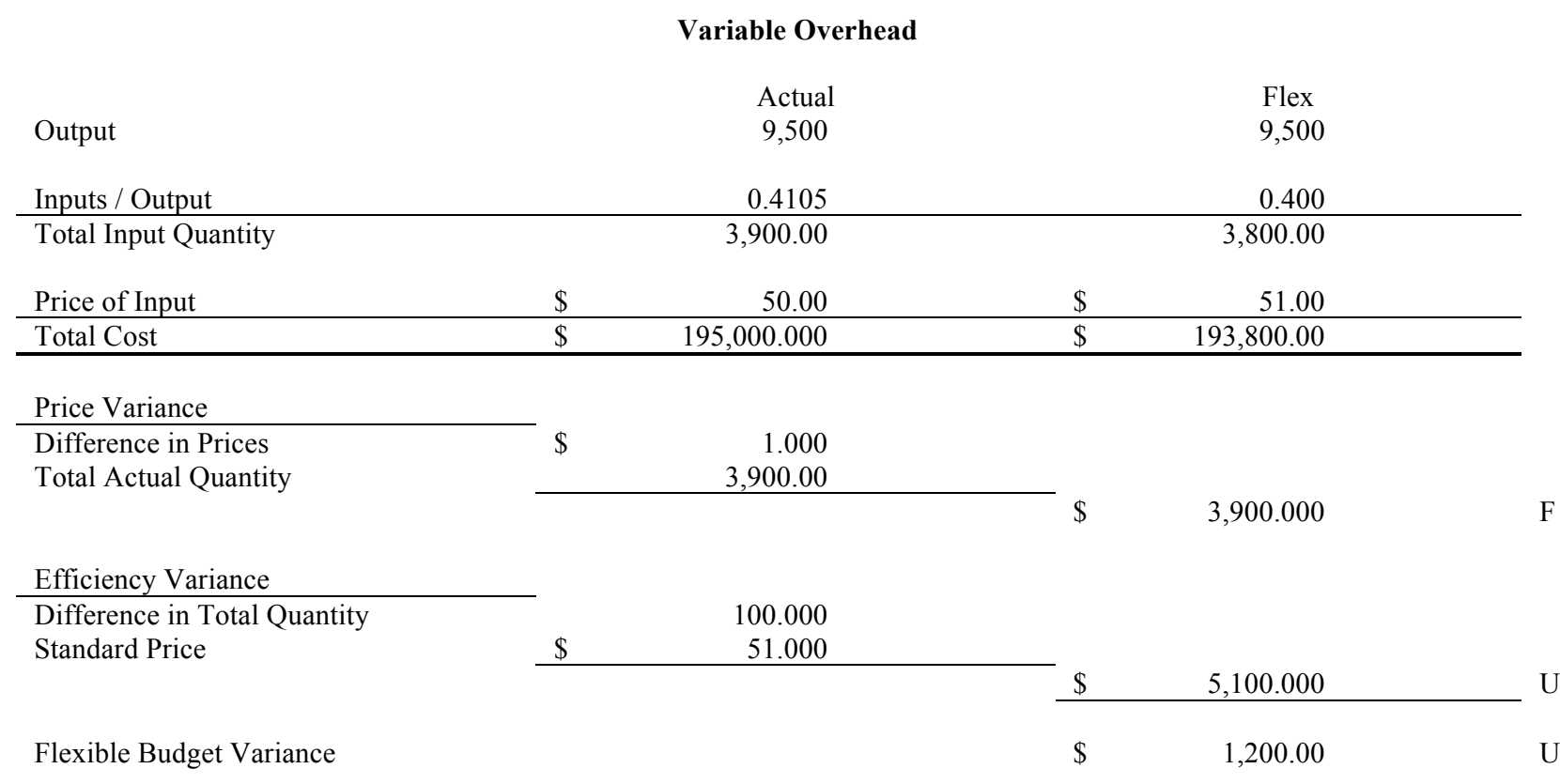

Fixed Overhead

$\begin{array}{ccc} & \text { Actual } & \text { Flex } \\ \text { Output } & 9,500 & 9,500\end{array}$

\begin{tabular}{lllll}
\hline Total Cost & $\$$ & $129,000.000$ & $\$$ & $130,000.00$ \\
Price Variance & & & & \\
\hline
\end{tabular}

$\$ \quad 1,000.000$

Efficiency Variance

Flexible Budget Variance

$\$$

$\$ \quad 1,000.000$

\section{SECTION 4: TEACHING NOTES AND SUGGESTIONS FOR THE INSTRUCTOR}

Cost accounting textbooks typically contain extensive technical coverage of standard costing and variance analysis in a manufacturing context. Covering the material adequately usually requires multiple class lectures, due to the complexity of the material. The calculations involved are detailed, multi-step, and can be numerically intensive. In order to make the material accessible to the learner, most textbook problems focus on the individual steps inherent in the budgeting, calculation, analysis, and interpretation process of standard costing and variance analysis. This simplification makes it feasible to deliver the material efficiently in the classroom. However, this pedagogical efficiency occurs at a cost, in that students may fail to understand the overall process and how the individual steps are interrelated. 
This case has been used over the course of 10 semesters with over 500 students in a junior level Cost Accounting class for accounting majors. It has proven to be an effective tool for both reinforcing the variance analysis content that is delivered in the classroom, as well as for giving students an opportunity to practice and extend their Excel skills.

A few additional details regarding the project appear below.

A. Timing of Assignment. Students seem to benefit most if this project is assigned at the beginning of the technical coverage of a Standard Costing, Flexible Budgeting, and Variance Analysis content module. The project can then be referenced and explained as each section of the technical material is covered.

B. Student Time to Complete. The project generally takes about 4 hours to complete, assuming that the students have a basic competence with Excel. This seems to hold true whether the project is done individually or in groups of two students.

C. Project Benefits for Students. As mentioned above, a strong educational benefit of this project is that it requires students to prepare an entire set of standard costing reports - beginning with the calculation of a Standard Cost and culminating in the generation of Price and Efficiency Variances. However, additional significant benefits include:

- Teaching the use of an Input Page that feeds the other worksheets,

- Forcing the extensive use of linking between and within worksheets in a workbook,

- Reinforcing basic Excel skills (summation, referencing, division, etc.),

- Introducing a few more advanced skills (Nested If Statements and Absolute Value Functions), and

- Emphasizing the importance of data organization in analysis and reporting.

\section{AUTHOR BIOGRAPHY}

Katherine J. Silvester, Ph.D. is an Associate Professor of Accounting at Siena College in Loudonville, NY. Prior to joining academia, she was employed in private industry for a decade in various management accounting roles. Her primary research interests include cost accounting, pricing, and accounting pedagogy. Her previous work has appeared in journals such as: The Journal of Accounting Case Research, The Journal of Higher Education Theory and Practice, and The Journal of Cost Management. E-Mail: ksilvester@siena.edu

\section{REFERENCES}

Horngren, C., Datar, S., \& Rajan, M. (2015). Cost accounting: A managerial emphasis, $15^{\text {th }}$ edition. Upper Saddle River, New Jersey: Pearson-Prentice Hall. 